\title{
Arp 220 - IC 4553/4: understanding the system and diagnosing the ISM
}

\author{
Willem A. Baan \\ ASTRON, Oude Hoogeveensedijk 4, 7991PD Dwingeloo, The Netherlands \\ email: baan@astron.nl
}

\begin{abstract}
Arp 220 is a nearby system in final stages of galaxy merger with powerful ongoing star-formation at and surrounding the two nuclei. Arp 220 was detected in HI absorption and $\mathrm{OH}$ Megamaser emission and later recognized as the nearest ultra-luminous infrared galaxy also showing powerful molecular and X-ray emissions. In this paper we review the available radio and mm-wave observational data of Arp 220 in order to obtain an integrated picture of the dense interstellar medium that forms the location of the powerful star-formation at the two nuclei.
\end{abstract}

Keywords. galaxies: ISM - galaxies: nuclei - ISM: molecules - ISM: masers - galaxies: starburst, - galaxies: OH Megamaser

\section{OH MM and ULIRGs}

The galaxy IC 4335, later referred to as Arp 220, was recognized early as a merging and strongly interacting system with a blue nucleus by Arp (1966) and Nilson (1973). The detection of strong HI absorption against the radio continuum of the nuclear starburst (Mirabel 1982) led to a search for corresponding $\mathrm{OH}$ absorption, which resulted in the detection of powerful $\mathrm{OH}$ maser emission later classified as $\mathrm{OH}$ MegaMaser emission (OH MM) (Baan et al. 1982). The extraordinary properties of Arp 220 were confirmed by the IRAS far-infrared (FIR) data (Sanders et al. 1988), which made Arp 220 the prototype Ultra-Luminous FIR Galaxy (ULIRG). The enhanced emission line strength of the molecular constituents were recognized and the CO lines were used to map the nuclear regions of ULIRGs (Solomon et al. 1997; Downes \& Solomon 1998; Sakamoto et al. 1999). More recently high-density molecular tracers have been studied in order to diagnose the molecular medium (Aalto et al. 2007; Baan et al. 2007; Graciá-Carpio et al. 2006; Greve et al. 2007).

Arp 220 experiences a powerful burst of starformation at each of the nuclei triggered by the ongoing merger, which results in the FIR prominence and multiple radio SNRs at each of the nuclei (Lonsdale et al. 2006) and SB-related hard X-ray emission (Iwasawa et al. 2005). Arp 220 is a recent example of a short-lived burst of assembly and nuclear activity that are common at redshifts of 2.5 and that defines the characteristics of massive galaxies in the nearby universe. Arp 220 is a template for high redshift active galaxies.

\section{Dynamics of Arp 220 and where is what?}

The two nuclei of Arp 220 are separated by only $0.97^{\prime \prime}$ (365 pc) as determined from their radio positions. They appear not yet severely deformed at this advanced stage of interaction and have a relatively small velocity difference. Therefore, the two mass centers are still sufficiently far away from each other and need to be one behind the other. Only the high-density tracer emission lines accurately trace the nuclear ISM of the two nuclei 


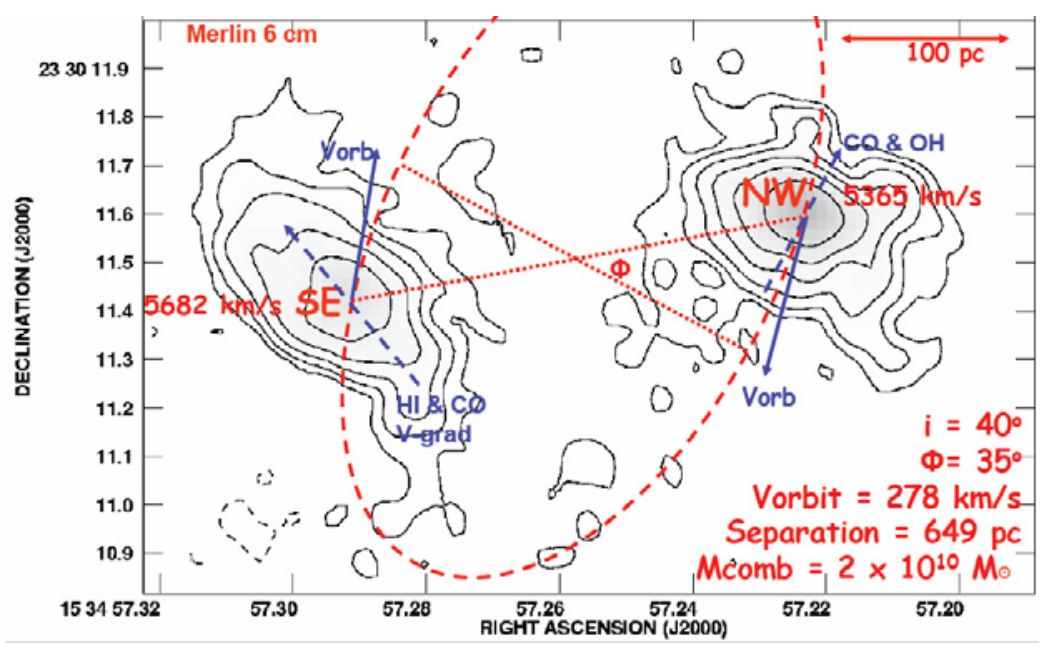

Figure 1. Orbital dynamics of Arp 220. The north-western nucleus is located in front of the south-eastern nucleus. The systemic velocities of the two equal-mass nuclei are indicated and their direction of motion in an orbital plane inclined by $40^{\circ}$. The orbital velocity $\left(V_{\text {orb }}\right)$ is $278 \mathrm{~km} \mathrm{~s}^{-1}$. The combined mass of the nuclei is based on an observed stellar and gas mass. The directions of the observed velocity gradients of the $\mathrm{CO}$ and $\mathrm{OH}$ molecular gas in the nuclei are indicated. The MERLIN 6cm continuum map is from Baan, Cohen \& Kl" ockner (2007).

having systemic velocities of $5365 \mathrm{~km} \mathrm{~s}^{-1}$ for the western nucleus and $5682 \mathrm{~km} \mathrm{~s}^{-1}$ for the eastern nucleus (see section 6).

The systemic velocities of the nuclear regions obtained from the high-density tracer emission lines may be used for dynamic modelling of the system. Similar models were presented earlier using $\mathrm{H}_{2} \mathrm{CO}(1-0)$ and $\mathrm{CO}(2-1)$ molecular data (Baan \& Haschick 1995; Downes \& Solomon 1998; Scoville et al. 1997). Reconsideration of the available continuum, $\mathrm{CO}, \mathrm{HI}$ absorption, and $\mathrm{OH}$ and $\mathrm{H}_{2} \mathrm{CO}$ emission data gives slightly different picture of the dynamics of the system (Baan, Cohen \& Kl"ockner 2007). The eastern nucleus lies behind the western nucleus, which would result in the observed larger column densities in front of the eastern nucleus (Iwasawa et al. 2005) and a relatively lower velocity for the western nucleus. Using the $\mathrm{CO}(2-1)$ signature, the orbital plane is inclined by $40^{\circ}$ (Scoville et al. 1997). The combined mass of lower-density molecular gas in CO, the high-density gas in tracers such as HCN, and the stellar component is of the order of 0.6 $-2.0 \times 10^{10} \mathrm{M}_{\odot}$ (Sakamoto et al. 1999; Greve et al. 2007). The dynamics of the system may follow from two equal-mass nuclei with a spatial separation of $650 \mathrm{pc}$ (Fig. 1).

Near-infrared imaging of Arp 220 using the NICMOS instrument $(1.6 \mu \mathrm{m})$ onboard Hubble ST confirm the presence of high column densities in the system (Scoville et al. 2000). The visual attenuation is 17 and 24 magnitudes for the East and West nuclei assuming a young stellar population and 13 and 20 magnitudes for an old population (note: one magnitude corresponds to $1.8 \times 10^{21} \mathrm{~cm}^{-2} \mathrm{H}$ ). The NIR emission actually peaks north of each of the nuclei; there are areas where virtually no radiation can escape, even at $2.2 \mu \mathrm{m}$. Reversing the symmetry of the model could place the eastern nucleus in front of the western nucleus, but would not explain the presence of $\mathrm{OH}$ emitting gas at the velocity of the western nucleus in front of the eastern nucleus (see section 4).

The hard X-ray $(3-7 \mathrm{KeV})$ signature of Arp 220 as observed by Chandra shows two separate (extended) nuclei and there is an extended nebula at $2 \mathrm{keV}$ (Iwasawa et al. 2005). The hard X-ray continuum is due to the integrated X-ray emission from binary systems and is a factor 10 too low for the observed SFR. There are plasma features 
associated with the ionized gas and a Fe $\mathrm{K} \alpha$ feature at $6.5 \mathrm{keV}$. The obscuration of the X-rays required $N_{H}=10^{22-23} \mathrm{~cm}^{-2}$ (Note: $N_{H}=10^{22} \mathrm{~cm}^{-2}$ is enough for $\mathrm{keV}$ absorption).

\section{Modelling the starburst}

The power generation for the FIR luminosity of $10^{12.2} \mathrm{~L}_{\odot}$ is dominated by starburst activity for more than $60 \%$ in the radio and more than $90 \%$ in the near-infrared (Baan \& Kl" ockner 2006; Genzel et al. 1998). The Arp 220 starburst is a scaled-up version of the M 82 activity where also Type II supernova and remnants of massive progenitor stars interact with the ISM. Modelling of the embedded starbursts suggests a star-formation rate $\mathrm{SFR}=26 \mathrm{~L}_{I R, 11} \mathrm{M}_{\odot} \mathrm{yr}^{-1}=340 \mathrm{M}_{\odot} \mathrm{yr}^{-1}$ and a supernova rate $\mathrm{SNR}=0.2 \mathrm{~L}_{I R, 11}$ $\mathrm{yr}^{-1}=2.8 \mathrm{yr}^{-1}$ for Arp 220 (Elson, Fall \& Freeman 1989). Lonsdale et al. (2006) have observed a SNR of $4 \pm 2 \mathrm{yr}^{-1}$ and find the rate in the western nucleus to be three times higher than in the eastern nucleus. The mass injection rate for the nuclei of Arp 220 is estimated at $53 \mathrm{M}_{\odot} \mathrm{yr}^{-1}$. Recent evaluation of the radio data suggests a top-heavy initial mass function (IMF) or a short starburst with a duration of $3 \times 10^{5}$ yr with a $\mathrm{SFR} \approx$ $10^{3} \mathrm{M}_{\odot} \mathrm{yr}^{-1}$ (Parra et al. 2007; Lonsdale et al. 2006).

The starburst in Arp 220 must be close to its peak in activity. Much of the molecular ISM appears still intact but in an excited state as evidenced from the warm high-density molecular material (see section 6 below). The line ratios of prominent high-density tracers suggest that the molecular ISM is (or is at the verge of) being depleted and destroyed by the ongoing star-formation process (see section 6 and Loenen, Baan \& Spaans 2007). There is evidence that destructive mechanical feedback due to supernovae and high-velocity outflows seen in $\mathrm{OH}$ emission are affecting the molecular ISM (section 7). Mechanical feedback due to a violent starburst and radiative feedback on the molecular chemistry could cut off the ongoing star formation, leaving a single (first) generation of stars with a top-heavy IMF.

\section{Imaging the $\mathrm{OH}$ emission lines}

Knowledge of the systemic velocities of the high-density gas at the two nuclei (see section 6) also helps to understand the complex $\mathrm{OH}$ groundstate line emission signature. Two pairs of (1665 and $1667 \mathrm{MHz}$ ) OH emission lines are offset by $317 \mathrm{~km} \mathrm{~s}^{-1}$ and have a $351 \mathrm{~km} \mathrm{~s}^{-1}$ separation within the pair. Therefore, triple emission lines are seen: the 1667 $\mathrm{MHz}$ West line, a convolved $1667 \mathrm{MHz}$ East and $1665 \mathrm{MHz}$ West line, and a $1665 \mathrm{MHz}$ East line. However, also emission in the $1667 \mathrm{MHz}$ line at the systemic velocity of West is seen at the East location (see VLA-A and VLBI data by Baan \& Haschick 1987; Rovilos et al. 2003). The gas responsible for this $1667 \mathrm{MHz}$ emission at the eastern nucleus cannot be foreground gas trailing from the western nucleus. This is also not solved by placing the eastern nucleus in the foreground in the dynamical picture of Fig. 1. The nature of all emissions and further verification of the dynamical scenario for the two nuclei may be deduced from a study of the lower-gain diffuse emission lines.

In addition to the three emission components due to two line pairs, there is a blueshifted component that is evidence for outflow in the nuclear region (Baan, Haschick \& Henkel 1989). The blue-shifted wing of the $1667 \mathrm{MHz}$ line in Arp 220 extends by $1145 \mathrm{~km} \mathrm{~s}^{-1}$, while the $1720 \mathrm{MHz}$ line extends by $950 \mathrm{~km} \mathrm{~s}^{-1}$, although this second outflow wing may be less-pronounced (Ghosh \& Salter 2007, personal communication).

The $\mathrm{OH}$ emission in Arp 220 at both nuclei encompasses the high-resolution radio continuum structure and the high-density ISM where the star-formation is taking place. 


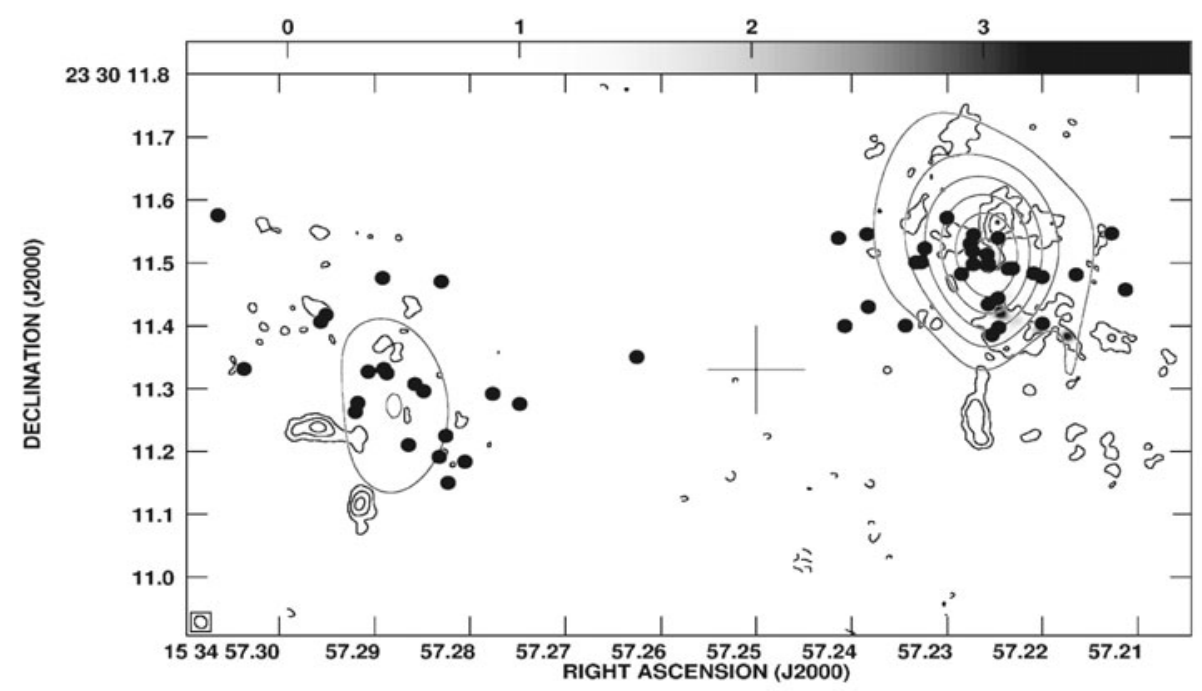

Figure 2. The $\mathrm{OH}$ emission at the two nuclei of Arp 220. The high-resolution EVN map of the $1667 \mathrm{MHz}$ emission from Rovilos et al. (2003) has been overlayed with the map of supernovae in the two nuclei from Parra et al. (2007). Continuum contours indicate the location of the radio nuclei. The superposition is a best effort. $\left(0.1^{\prime \prime}=35 \mathrm{pc}\right)$.

At MERLIN resolution $\left(0.17^{\prime \prime}=61 \mathrm{pc}\right)$ the $1667 \mathrm{MHz} \mathrm{OH}$ emission at the eastern nucleus is centrally peaked and has an extent of $170 \mathrm{pc}$ along the velocity gradient (Rovilos et al. 2003), which agrees with the extent of the high-brightness components found at EVN resolution $\left(0.016^{\prime \prime}\right)$ (see Fig. 2). Similarly the emission at the Western nucleus shows a extended $\left(1.2^{\prime \prime}=420 \mathrm{pc}\right)$ double (torus-like) structure straddling the continuum nucleus (Fig. 2; Rovilos et al. 2003) with clumpy high-brightness components only in the central $0.6^{\prime \prime}$ region. At both nuclei the elongated/edge-on distribution of high-brightness $\mathrm{OH}$ components extends beyond the starburst-related cluster of supernova remnants in the continuum (Rovilos et al. 2005; Lonsdale et al. 2006; Parra et al. 2007). The OH velocity gradients at both nuclei are similar to those in the $\mathrm{CO}(2-1)$ data (see Fig. 1), which would confirm the presence of orbiting counter-rotating disks (Sakamoto et al. 1999).

The $\mathrm{OH}$ characteristics suggest that the emissions at both nuclei originate both in the nuclear ISM and in an edge-on torus/disk structure that surrounds the nuclear ISM with the ongoing star formation. At the Eastern component the amplifying medium of the major emission component is the (foreground) frontal section of the torus at the systemic velocity. The double structure of the Western component defines the two edge-sections of a similar thick torus with radius of about $60 \mathrm{pc}$. The high-brightness components emission components primarily trace structures in the nuclear region and result from spatial superpositions of clouds and (edge-on) shocked regions associated with the star formation. OH emission is also seen in front of individual SNR remnants (see Lonsdale et al., these proceedings). The edge-on torus may serve for further amplification. Successful modelling of observed $\mathrm{OH}$ emission structures has been done for Mrk 273 and IIIZw 35 (Parra et al., these proceedings; Kl"ockner 2004; Parra et al. 2005).

\section{Clumpy low-gain amplification for $\mathrm{OH}$}

The standard model for masers of low-gain amplification of radio continuum by foreground molecular material has been suggested in order to explain the extremely powerful 
emissions in OH MM (Baan 1985, 1989; Henkel \& Wilson 1990). The spatial distribution of the background radio continuum and the availability of a pumping agent will allow a foreground screen to selectively amplify the distributed continuum. Assuming a uniform screen gives a first order estimate of the global amplification. A more realistic nonuniform and clumpy nuclear ISM will give non-uniform amplification in high-resolution data. Emission is found in all $\mathrm{OH}$ ground state transitions in Arp 220, while the known 5 $\mathrm{GHz}$ and $6 \mathrm{GHz}$ lines are all in absorption in both $\mathrm{OH}$ MMs and absorbers (Henkel et al. 1987; Impellizzeri et al. 2005). An amplification scenario is relevant for extragalactic and also galactic emissions of $\mathrm{OH}, \mathrm{H}_{2} \mathrm{CO}, \mathrm{H}_{2} \mathrm{O}$, and $\mathrm{CH}_{3} \mathrm{OH}$.

Evaluation of the high-resolution data suggests a gain factor of 4 to 5 relative to the background $(\tau=1.4-1.6)$ for the extended components that account for the one third of the $1667 \mathrm{MHz}$ emission (Rovilos et al. 2003). The compact components have a gain factor ranging from $46-65$ at the southern part of the western nucleus to 125 at the northern part $(\tau=3.8-4.8)$. Clump superpositions or edge-on (shocked) shells need to increase the optical depth by a factor up to three.

Pumping scenarios for the OH molecules (Henkel et al. 1987; Randell et al. 1995; Lockett \& Elitzur 2007; Parra et al. 2005, and Elitzur, these proceedings) provide specific requirements for the physics of the ISM of the galactic nuclei. The efficiency of the radiative pumping using the 53 and $35 \mu \mathrm{m}$ FIR lines puts requirements on the FIR-SED and requires a threshold temperature for the dust of at least $50 \mathrm{~K}$, whereas Arp 220 has $61 \mathrm{~K}$. Typically clouds of $1 \mathrm{pc}$ should have an opacity of one and a linewidth of $10 \mathrm{~km} \mathrm{~s}^{-1}$ for line-overlap conditions. Pumping can be achieved across a density range of $\mathrm{n}\left(\mathrm{H}_{2}\right)=10^{3}$ to few $10^{4} \mathrm{~cm}^{-3}$. For $T_{e x}(O H)$ approximately constant, the line ratios are a function of optical depth and give an optical depth of order 2.0, which is consistent with observations. Simulations of the cloud superposition and the amplification in a torus-like structure has been done by $\mathrm{Kl}^{\prime \prime}$ ockner (2004) and Parra et al. (2005).

\section{The high-density ISM}

Diagnostics of the heavily obscured nuclear ISM can also be done by interpreting molecular tracers to determine densities, temperatures and the molecular chemistry. Accurate estimates of global (integrated) properties can be obtained with LVG (large velocity gradient) radiation transfer studies using line emission along the energy ladder of the molecule. In addition, the chemical evolution of molecular species combined with their radiative transfer can be obtained by modelling X-ray and Photon (UV) dominated regions (XDR and PDR) (Meijerink, Spaans \& Israel 2006, 2007, also Spaans et al. and Loenen et al., these proceedings).

Many molecular transitions have been detected in Arp 220 and in similar nearby (U)LIRGs like NGC 6240, and Mrk231. Single-dish spectra obtained with the IRAM $30 \mathrm{~m}$ and SEST $15 \mathrm{~m}$ telescopes of some of the tracer lines in Arp 220 are presented in Figure 3 (Baan et al. 2007). Two features can be seen in Fig. 3 with systemic velocities of 5365 and $5683 \mathrm{~km} \mathrm{~s}^{-1}$ for the western and eastern nuclei (Baan et al. 2007). Higher transitions have been detected for $\mathrm{CO}, \mathrm{CS}, \mathrm{CN}, \mathrm{HCN}, \mathrm{HNC}$, and $\mathrm{HCO}^{+}$(Aalto et al. 2007; Graciá-Carpio et al. 2006; Greve et al. 2007; Wiedner et al. 2002). Recent maps of the prominent $\mathrm{HCN}$ (4-3) line at $270 \mathrm{GHz}$ display localized emission with a spatial extent similar to that of the cluster of discrete radio sources (Wiedner et al. 2007).

The molecular ISM in ULIRGs is expected to have extreme excitation conditions where the physical environment and the molecular chemistry have been affected by the radiation fields and the feedback from the star-formation process. The environment is very different from those in Galactic SF regions. The interpretation of extragalactic phenomena requires 

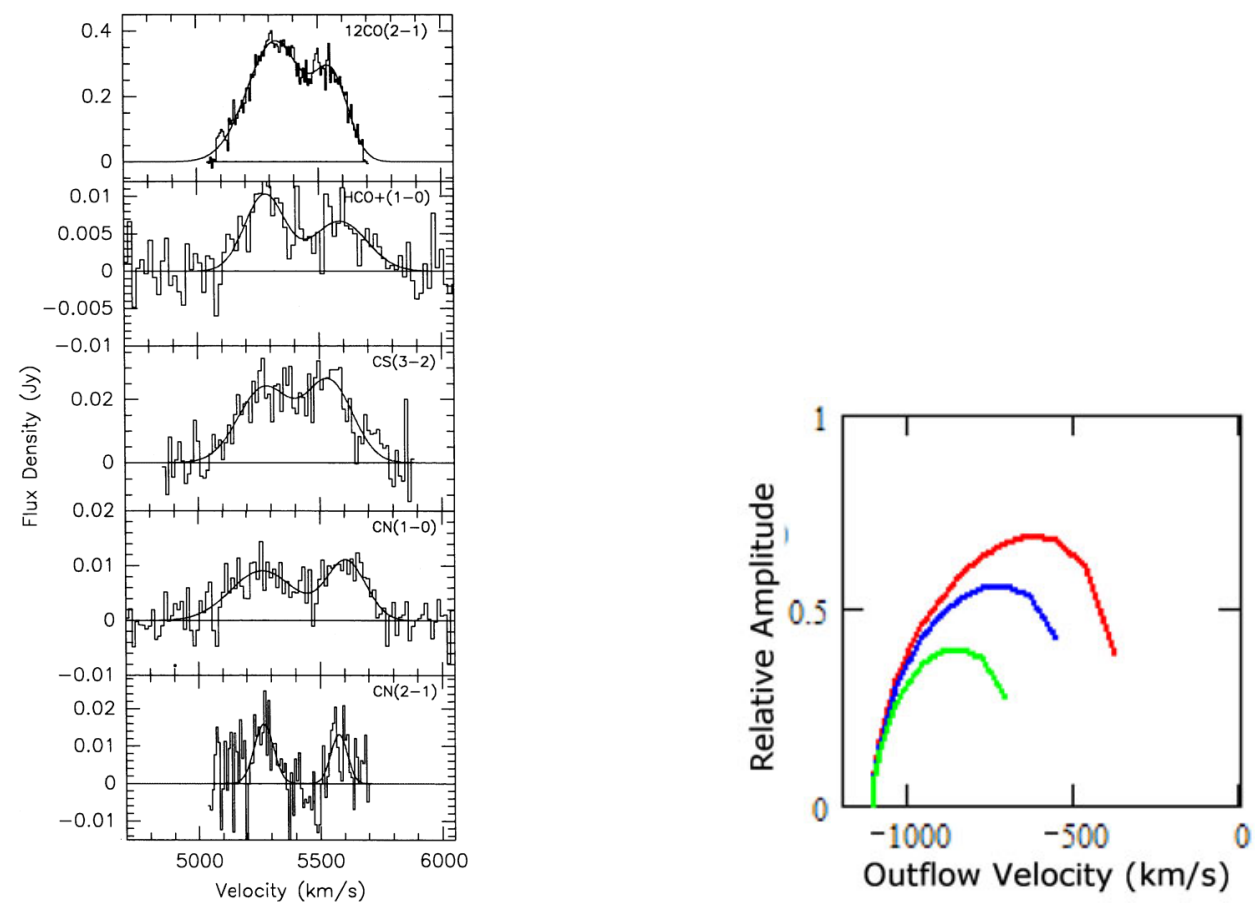

Figure 3. Spectra of emission lines of high-density tracer molecules in Arp 220. From top to bottom the emission line spectra of $\mathrm{CO}(2-1), \mathrm{HCO}^{+}(1-0), \mathrm{CS}(3-2), \mathrm{CN}(1-0)$, and $\mathrm{CN}(2-1)$. Data taken from Baan et al. (2007).

Figure 4. Simulations of the amplified $\mathrm{OH}$ emission from outflows driven by supernova remnants. The three curves result from different covering factors for the spherical layers of molecular gas relative to the radio continuum generated by the SNR. The chosen terminal velocity is as observed in Arp 220.

an extrapolation of well-resolved Galactic phenomena to integrated and unresolved nuclear environments. High-density components may be used to discriminate between PDR and XDR excitation conditions (Baan et al. 2007). In particular, the HNC/HCN ratio has values less than unity in PDRs and values larger than unity in XDRs. Similarly the ratios of $\mathrm{HCO}^{+}$with $\mathrm{HCN}$ and $\mathrm{HNC}$ discriminate between the gas density due to different critical densities of the molecules. The intensity ratio of a high-density tracer molecule with that of a lower density tracer, such as CO (1-0) that is more extended and is less affected by the star-formation activity, shows that the high-density medium varies strongly during the lifetime of the activity (Baan et al. 2007). The extreme molecular ratios for Arp 220 and those of other OH MM suggest extreme PDR-like conditions for the nuclear ISM (Loenen et al. these proceedings and Loenen, Baan \& Spaans 2007).

LVG simulations suggest a two-phase ISM for the combined nuclei of Arp 220 with a diffuse component with $\mathrm{n}\left(\mathrm{H}_{2}\right)=2 \times 10^{3} \mathrm{~cm}^{-3}$ and $\mathrm{T}_{k}=40-60 \mathrm{~K}$ and dense components with $\mathrm{n}\left(\mathrm{H}_{2}\right)=10^{4-6} \mathrm{~cm}^{-3}$ and $50-70 \mathrm{~K}$ (Greve et al. 2007). The molecular gas is contained within self-gravitating and virialized clouds with mass $0.6-2.0 \times 10^{10} \mathrm{M}_{\odot}$. The considerably higher pressure in the warm high-density medium as compared with the low-density medium will gradually cause disruption of the medium. 


\section{Signature of nuclear outflows}

Arp 220 is one of the $\mathrm{OH}$ MM galaxies showing blueshifted outflow components for their $1667 \mathrm{MHz}$ emission lines (Baan, Haschick \& Henkel 1989; Baan 2007). The observed outflow velocities vary with $L_{F I R}$ and are likely associated with the shocked shells surrounding supernova remnants. A continuing high-intensity starburst as found in such ULIRGs would eventually result in merging the localized outflows into a largescale blowout or nuclear superwind Heckman, Armus \& Miley (1990). The OH outflow emission results from a superposition shocked SNR shells propagating into the dense ISM that amplify the embedded radio continuum. A simple simulation of a superposition of superposed spherical outflowing shells amplifying a spherical continuum structure provides the correct shape and signature of the outflow (see Figure 4). The observed high velocities of the outflows suggest that J-shock chemistry is responsible for the molecular abundances. Molecules are destroyed and reassembled in J-shocks for $\mathrm{V}>50 \mathrm{~km} \mathrm{~s}^{-1}$ while for $\mathrm{V}>300 \mathrm{~km} \mathrm{~s}^{-1}$ this reassembly process happens at a slower rate. Using an energy conserving scenario for the mechanical energy (see Elson, Fall \& Freeman 1989), we find that: $\mathrm{N}_{S N} \mathrm{R}_{k p c}^{2} \mathrm{~V}_{100}^{3} \mathrm{n}_{I S M} \approx 23 \mathrm{~L}_{I R, 11}$, which balances the total energy of the outflows from $\mathrm{N}_{S N}$ SNRs with outflow velocity $\mathrm{V}$ (in units $100 \mathrm{~km} \mathrm{~s}^{-1}$ ) and with radius $\mathrm{R}$ (in kpc) and the injected energy $\mathrm{L}_{I R}$ (in units of $10^{1} 1 \mathrm{~L}_{\odot}$ ). Using $\mathrm{L}_{I R}=1.6 \times 10^{12} \mathrm{~L}_{\odot}$ for Arp 220, an observed number of $\mathrm{N}_{S N} \approx 100$, an observed maximum velocity $\mathrm{V}(\max )$ $=1150 \mathrm{~km} \mathrm{~s}^{-1}$, and a typical SNR radius $=1 \mathrm{pc}$, we find that the mean ambient density of he pre-shock material $\mathrm{n}_{I S M}=1.5 \times 10^{3} \mathrm{~cm}^{-3}$, which is in an acceptable range. The interpretation of these outflows clearly requires more simulations as it provides further evidence of the eruptive nature of the star-forming environment.

\section{Formaldehyde emission}

Formaldehyde emission has been verified in Arp 220 as well as two other OH MM, and an $\mathrm{H}_{2} \mathrm{O}$ MM using the Arecibo and Effelsberg telescopes (Baan, Haschick \& Uglesich 1993; Araya, Baan \& Hofner 2004). At low resolution the formaldehyde in Arp 220 peaks at the western nucleus and has weak emission towards the eastern nucleus and the connecting structure (Baan \& Haschick 1995). Recent MERLIN data shows an elongated emission region of $80 \mathrm{pc}$ at the western nucleus (resolution $0.04 "=15 \mathrm{pc}$ ) centered at $5325 \mathrm{~km} \mathrm{~s}^{-1}$, which is $40 \mathrm{~km} \mathrm{~s}^{-1}$ below the systemic velocity of the western nucleus (Baan, Cohen \& $\mathrm{Kl}^{\prime \prime}$ ockner 2007). The velocity gradient of $\mathrm{H}_{2} \mathrm{CO}$ aligns with the weak gradient of the $\mathrm{OH}$ emission.

The diagnostics of this emission is still limited by the understanding of the pumping mechanism. Because the formaldehyde emission at lower resolution mimics the $2.2 \mu \mathrm{m}$ emission, the IR radiation field could play a role in the pumping. A first pumping model proposed for the excitation of the extragalactic formaldehyde was based on pumping by the non-thermal radio continuum, which gives small (few percent) optical depths for densities of $10^{2-5} \mathrm{~cm}^{-3}$ (Baan, $\mathrm{G}^{\prime \prime}$ usten \& Haschick 1986). While this model could operate in extragalactic environments, it poses a problem for small scale Galactic environments. Evaluation of collisional and other pumping schemes for Galactic and extragalactic environments shows that optical depths at the relevant density range are still relatively small and may not yet satisfy maser requirements under Galactic conditions (see Araya, Baan \& Hofner 2004; Baan, Haschick \& Uglesich 1993; Araya et al. 2007, and Darling, these proceedings). The high- and low-resolution formaldehyde data suggests that emission may be tracing high- and low-density gas components in a larger (foreground) gas structure that is not confined to the site of the star-formation itself. 


\section{Conclusions}

Radio observational tools contribute significantly to the understanding of the nuclear medium of Arp 220. Arp 220 is dynamically advanced such that the two nuclei have developed a common gas/stellar structure in advance of the merger. The current dynamical picture can be determined on the basis of the observed velocities of the two nuclear ISM components. Placing the eastern nucleus, with the highest obscuration, behind the western nucleus on a (first approximation) circular orbit explains the large-scale characteristics of the system but it does not yet explain $\mathrm{OH}$ emission at discrepant velocities and the extended continuum structure between the nuclei (see Rovilos et al. 2003).

The observed molecular components in Arp 220 sample a density range from $10^{2}$ to $10^{6} \mathrm{~cm}^{-3}$. The low-density medium with $10^{2-3} \mathrm{~cm}^{-3}$ is traced by the extended diffuse and clumpy CO components. The CO (2-1) transition traces the upper end of this range in nuclear regions enveloping the highest density medium. The high-density constituents have densities in the range $10^{4}$ to $10^{6} \mathrm{~cm}^{-3}$ depending on the critical density of the molecules. LVG simulations suggest that the temperature of the low-density tracer emissions ranges from 40-60 K, while those of the higher density components suggest $50-70 \mathrm{~K}$. This overpressure of the high-density components results from radiative and mechanical feedback of the star-formation process. The integrated dust temperature from the FIR SED is about $T_{d}=40 \mathrm{~K}$, while the local conditions in Arp 220 with $T_{d}>60 \mathrm{~K}$ provide the required pumping photons for the $\mathrm{OH}$ emission.

Both nuclear regions harbor a powerful and rapidly evolving starburst at the point destroying its own ISM. The star-formation rate is on the order of $340 \mathrm{M}_{\odot} \mathrm{yr}^{-1}$ and occurs predominantly in the western nucleus. The ISM in these nuclei is still mostly intact but there is evidence of radiative and mechanical feedback at work. The overpressure of the high-density molecular constituents and the observed $\mathrm{OH}$ outflows will in time redistribute and homogenize the nuclear ISM. Radiative feedback also results in a PDR dominated molecular chemistry. The effects of feedback on the molecular medium and the star-formation process would lead to a top-heavy (truncated) IMF and lead to the early termination of the star-formation. There is no evidence for the presence of an AGN in the nuclear regions and any associated XDR conditions. In addition, the observed emission due to X-ray binaries is too weak for the level of star-formation activity. The nuclei of Arp 220 have become the prototype of an extreme nuclear environment with dominant PDR conditions.

The FIR-pumped $\mathrm{OH}$ emission extends beyond the nuclear activity regions and shows diffuse structures as well as clumpy and shell-like components with densities of $10^{2-4} \mathrm{~cm}^{-3}$ (see Fig. 2 Rovilos et al. 2003). The diffuse and clumpy $\mathrm{H}_{2} \mathrm{CO}$ can be collisionally or radiatively pumped for densities of $10^{3-5} \mathrm{~cm}^{-3}$. The SNR-driven shells seen in the $\mathrm{OH}$ emission are not yet well-constrained but ambient densities of $10^{3} \mathrm{~cm}^{-3}$ are consistent. The diffuse and the high-brightness $\mathrm{OH}$ components cover regions with sizes of 1 to $100 \mathrm{pc}$, and result from a distributed clumpy molecular medium contained in two edge-on thick tori with a radius of about $60 \mathrm{pc}$ that surround the nuclear ISM at each nucleus. The highest amplifying gains result from cloud superpositions and edge-on shells (also seen as outflows) in the nuclear ISM. The $\mathrm{OH}$ and $\mathrm{H}_{2} \mathrm{CO}$ emission structures trace intermediatedensity components surrounding the highest density ISM structures co-located with the sites of star-formation. The special conditions leading to $\mathrm{OH}$ and $\mathrm{H}_{2} \mathrm{CO} \mathrm{MM}$ activity may occur during a significant part of the duration of the starburst.

The high-resolution VLBI data on the $\mathrm{OH}$ and $\mathrm{H}_{2} \mathrm{CO}$ emissions and the data for highdensity molecular tracers provide a complementary picture of the nuclear ISM and the ongoing SB activity in Arp 220. While the emission of the high-density tracer molecules presents the integrated properties of the nuclear ISM and is mostly unresolved at the 
two nuclei, the high-resolution data on the $\mathrm{OH}$ and $\mathrm{H}_{2} \mathrm{CO}$ emissions provides sufficient structural detail of the medium surrounding the sites of star formation. The interpretation of integrated properties depends on our ability to extrapolate from the understanding of Galactic environments to the extreme case of unresolved nuclear environments in starburst nuclei.

\section{References}

Aalto, S., Spaans, M., Wiedner, M. C. \& H"uttemeister, S. 2007, A\&AA, 464, 193

Araya, E., Baan, W. A. \& Hofner, P. 2004, ApJS, 154, 541

Araya, E., et al. 2007, ApJ, submitted

Arp, H. 1966, ApJS, 14, 1

Baan, W. A. 1985, Nature, 315, 26

Baan, W. A. 1989, ApJ, 338, 804

Baan, W. A. 2007, New Astron. Rev., 51, 149

Baan, W. A., Cohen, R. J. \& Kl" ockner, H.-R. 2007, in preparation

Baan, W. A. \& Haschick, A. D. 1987, ApJ, 318, 139

Baan, W. A. \& Haschick, A. D. 1995, ApJ, 454, 745

Baan, W. A., Haschick, A. D. \& Henkel, C. 1989, ApJ, 346, 680

Baan, W. A., Haschick, A. D. \& Uglesich, R. 1993, ApJ, 415, 140

Baan, W. A., Henkel, C., Loenen, A. F., Baudry, A. \& Wiklind, T. 2007, A\&A, in the press

Baan, W. A., G" usten, R. \& Haschick, A. D. 1986,

Baan, W. A. \& Kl" ockner, H.-R. 2006, A\& A, 449, 559

Baan, W. A., Wood, P. A. D. \& Haschick, A. D. 1982, ApJL, 260, L49

Downes, D. \& Solomon, P. 1998, ApJ, 507, 615

Elson, R. A. W., Fall, S. M. \& Freeman, K. C. 1989, ApJ, 336, 734

Genzel, R. et al. 1998, ApJ, 498, 579

Greve,T., Papadopoulos, P., Gao, Y. \& Radford, S. 2007, ApJ, submitted

Graciá-Carpio, J., García-Burillo, S., Planesas, P. \& Colina, L. 2006, ApJ, 640, L135

Heckman, T., Armus, L. \& Miley, G. 1990, ApJS, 74, 833

Henkel, C. \& Wilson, T. L. 1990, A\&SA, 229, 431

Henkel, C., G" usten, R. \& Baan, W. A. 1987, A\& A, 185, 14

Impellizzeri, C. M. V., Roy, A. L., Henkel, C. et al. 2005, Astron. Nachr., 326, 544

Iwasawa, K., Sanders, D. B., Evans, A. S., et al. 2005, MNRAS, 357, 565

$\mathrm{Kl}^{\prime \prime}$ ockner, H.-R. 2004, PhD thesis, University of Groningen

Lockett, P. \& Elitzur, M. 2007, in preparation

Loenen, A. F., Baan, W. A. \& Spaans, M. 2007, in preparation

Lonsdale, C. J., Diamond, P. J., Thrall, H., Smith, H. E. \& Lonsdale, C. J. 2006, ApJ, 647, 185

Meijerink, R., Spaans, M. \& Israel, F. 2006, ApJL, 650, L103

Meijerink, R., Spaans, M. \& Israel, F. 2007, $A \& A$, 461, 793

Mirabel, F. I. 1982, ApJ, 260, 75

Nilson, P. 1973, Nova Acta Regiae Soc. Sci. Upsaliensis, Ser. V, 0

Parra, R., Conway, J. E., Elitzur, M. \& Pihlstr"om, Y. M. 2005, A $\& A, ~ 443,383$

Parra, R., Conway, J. E., Diamond, P. J., et al. 2007, ApJ, 659, 314

Randell, J., Field, D., Jones, K. N., Yates, J. A. \& Gray, M. D. 1995, A\&AA, 300, 659

Rovilos, E., Diamond, P. J., Lonsdale, C. J., et al. 2003, MNRAS, 342, 373

Rovilos, E., Diamond, P. J., Lonsdale, C.J. et al. 2005, MNRAS, 359, 827

Sakamoto, K., Scoville, N. Z., Yun, M.S., et al. 1999, ApJ, 514, 68

Sanders, D. B., Soifer, B. T., Elias, J. H. et al. 1988, ApJ, 325, 74

Scoville, N. Z., Evans, A. S., Thompson, R., et al. 2000, ApJS, 119, 991

Scoville, N. Z., Yun, M.S., \& Bryant, P. M. 1997, ApJ, 484, 702

Solomon, P., Downes, D., Radford, S. J. E. \& Barrett, J. W. 1997, ApJ, 478, 144

Wiedner, M. C., Wilson, C. D., Harrison, A., et al. 2002, ApJ, 581, 229

Wiedner, M., Aalto, S., Volgenau, N. H. et al. 2007, in preparation 